06

\title{
Самоорганизация разномасштабных структурных групп в малоуглеродистой проволоке под действием интенсивного волочения
}

\author{
(C) З.А. Самойленко, ${ }^{1}$ Н.Н. Ивахненко, ${ }^{1,2}$ Е.И. Пушенко, ${ }^{1}$ В.Н. Варюхин, ${ }^{1}$ Е.Г. Пашинская, ${ }^{1}$ \\ В.М. Ткаченко, ${ }^{1}$ А.В. Завдовеев ${ }^{1}$ \\ ${ }^{1}$ Донецкий фризико-технический институт им. А.А. Галкина, \\ 83000 Донецк, Украина \\ ${ }^{2}$ Донецкий национальный университет экономики и торговли им. М. Туган-Барановского, \\ 83050 Донецк, Украина \\ e-mail: yulduz19.77@mail.ru
}

(Поступило в Редакцию 31 октября 2017 г.)

Исследованы особенности механических параметров и атомной структуры проволок из стали 08Г2С под действием интенсивного волочения с комбинированием сдвиговой волоки с классическими круглыми волоками разного диаметра. Расщепленная форма интерференционных максимумов (211) на рентгенограммах образцов свидетельствует о расслоении в семействах указанных плоскостей на сжатые и растянутые группы, которое сопровождается частичным фазовым переходом порядок-беспорядок с образованием аморфизированной фракции из фрагментов наноразмерных атомных группировок этих плоскостей (кластеров). В зависимости от деформации стали выявлен характер изменения симметрии распределения структурных групп относительно углового положения дифракционного максимума от плоскостей (211). Установлено, что при увеличении деформации $(\varepsilon>30 \%)$ интенсивности когерентного и некогерентного рентгеновского $\mathrm{Cr}$ $\mathrm{K} \alpha$-излучений количественно изменяются, что доказывает развитие процессов самоорганизации, способствующих увеличению объемной доли порядка относительно беспорядка в стали 08Г2С, даже подвергаемой сильному механическому воздействию.

DOI: 10.21883/JTF.2019.02.47074.2538

\section{Введение}

В настоящее время для получения материалов с ультрамелкозернистой структурой используются как отдельные методы интенсивной пластической деформации со сдвигом, такие как, например, равноканальное угловое прессование, винтовая экструзия, кручение под высоким давлением [1-4], так и их комбинации с последующей прокаткой, осадкой, волочением и др. [5-11]. Применение этих методов позволяет значительно повысить прочность материала при сохранении определенного запаса пластичности. Однако объемы материалов, получаемые с помощью вышеперечисленных методов интенсивной пластической деформации, не всегда достаточно велики по сравнению с необходимыми для производственных масштабов [5-8], или применяются конструктивно сложные деформирующие блоки [10,11]. Для получения проволочных длинномерных изделий с высоким комплексом механических свойств была предложена технология волочения со сдвигом [12,13], применение которой приводит к увеличению прочностных свойств с одновременно повышенным запасом пластичности, что позволяет удешевить и упростить технологию производства за счет упразднения процесса промежуточного отжига. Применение сдвиговой волоки приводит к росту микротвердости. С увеличением степени обжатия сдвиговой волокой наблюдается тенденция к уменьшению анизотропии микротвердости в продольном и поперечном сечении. Это, безусловно, положительное явление, так как вызывает увеличение ресурса пластичности проволоки. С целью изучения механизмов структурообразования при такой обработке актуальным является исследование изменений, происходящих в тонкой структуре стали 08Г2С при интенсивной деформации во время волочения. Как было показано, в многоатомной конденсированной системе, подвергаемой сильному механическому воздействию в результате комбинированного волочения со сдвиговой волокой и затем со стандартными круглыми волоками разного диаметра, происходит развитие процессов самоорганизации, при этом для сильно деформированного материала энергетически выгодной является предпочтительность разномасштабной фрагментации атомной структуры при компактировании групп плоскостей либо в состоянии растяжения при деформации образца $(\varepsilon=39.6 \%)$, либо же в состоянии сжатия при деформации $(\varepsilon=46 \%)$.

\section{1. Материал и методики эксперимента}

Исследовали изменения атомного порядка структуры стали 08Г2С (см. таблицу) после комбинированной обработки, заключающейся в волочении по новой экспериментальной технологии со сдвиговыми волоками и классической технологии со стандартными круглыми волоками. Волочение осуществляли на стане 
Химический состав проволоки из стали 08Г2С, \%

\begin{tabular}{c|c|c|c|c|c|c|c|c}
\hline $\mathrm{C}$ & $\mathrm{Mn}$ & $\mathrm{Si}$ & $\mathrm{S}$ & $\mathrm{P}$ & $\mathrm{Cr}$ & $\mathrm{Ni}$ & $\mathrm{Cu}$ & $\mathrm{N}$ \\
\hline 0.071 & 1.98 & 0.84 & 0.015 & 0.018 & 0.015 & 0.009 & 0.016 & 0.0055 \\
\hline $\mathrm{Ti}$ & $\mathrm{As}$ & $\mathrm{B}$ & $\mathrm{Al}$ & $\mathrm{V}$ & $\mathrm{Mo}$ & $\mathrm{W}$ & $\mathrm{Co}$ & \\
\hline$<0.005$ & $<0.005$ & $<0.0005$ & 0.005 & 0.006 & $<0.01$ & 0.024 & 0.01 &
\end{tabular}

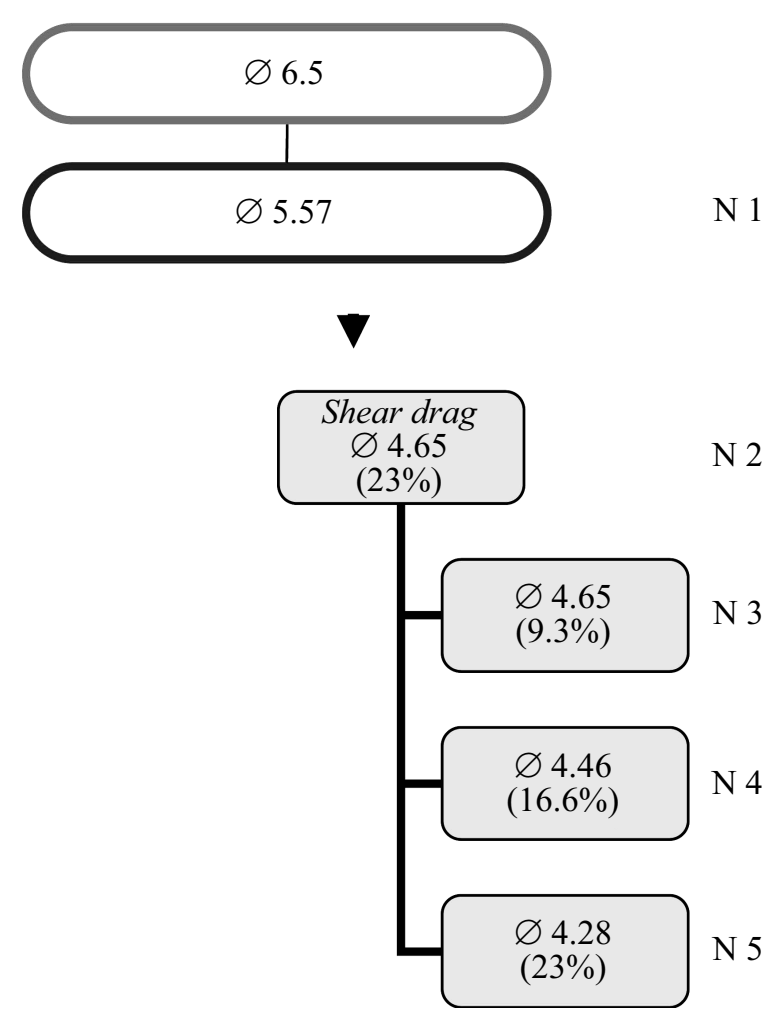

Рис. 1. Схема волочения, в процентах приведены разовые обжатия и номера образцов: № $1, \varepsilon=0$; № $2, \varepsilon=23 \%$, сдвиговая волока; № 3, сдвиговая волока + круглая волока $\varepsilon=23+9.3=32.3 \%$; № 4, сдвиговая волока + круглая волока, $\varepsilon=23+16.6=39.6 \%$; № 5, сдвиговая волока + круглая волока $\varepsilon=23+23=46 \%$.

АЗТМ 7000/1. Смазка подсыпалась на каждом этапе волочения. Скорость волочения - $1.1 \mathrm{~m} / \mathrm{s}$. Эксперимент был построен следующим образом (рис. 1):

1) из катанки ф6.5 проведено волочение обычным способом для получения круглого диаметра ф 5.57;

2) проведен отжиг полученного образца при $650^{\circ} \mathrm{C}$ и $30 \mathrm{~min}$ выдержке, охлаждение проводилось на воздухе (образец № 1, исходное состояние $\varepsilon=0$ );

3) полученный типоразмер волочили через волоку со сдвигом ф4.65 (образец № 2, $\varepsilon=23 \%$ );

4) типоразмер разделили на три части и проволочили через круглую волоку ф 4.65, ф4.46, ф 4.28. Это дало три варианта состояний с разными частичными обжатиями на первом переходе, обычная волока - волока со сдвигом, $\varepsilon=0 \rightarrow 23 \%$, и последующих трех переходах, сдвиговая волока - волока обычная: $\varepsilon=23+9.3 \%$, образец № $3 ; \varepsilon=23+16.6 \%$ - № 4 и $\varepsilon=23+23 \%$ № 5. В образцах № 3-5 первая деформация обусловлена сдвиговой волокой, а вторая деформация - обычной, круглой.

На образцах измеряли механические свойства, в частности, предел прочности и относительное сужение. Механические испытания на прочность выполняли на машине УММ-50 при температуре $293 \mathrm{~K}$ и скорости нагружения $10 \mathrm{~mm} / \mathrm{min}$ согласно ГОСТ 25.601-80.

Микроструктуру отожженного и деформированных образцов изучали при увеличениях 100-1000 на прибоpe „Neophot-32“ после многократной полировки и травления на границы зерен (состав травителя: 4\% азотной кислоты, 96\% спирта). Фотографирование осуществляли на оптическом микроскопе Axiovert 40 МАТ с помощью программного обеспечения Axiovision 4.6.

Для анализа атомной структуры дальнего и мезоскопического порядков были получены дифракционные картины от изучаемых образцов стали 08Г2С в $\mathrm{Cr} \mathrm{K} \alpha$ излучении с $V$-фильтром фотометодом, чувствительным к регистрации диффузного когерентного и некогерентного рассеяний рентгеновских лучей, что позволяет анализировать детали структурных изменений атомного порядка в случае сильных механических воздействий [14-18].

\section{2. Результаты эксперимента и обсуждение}

\section{1. Различие в свойствах прочности и пластичности образцов}

Подготовленные образцы № 1-5 были исследованы на прочность и пластичность. Для исследуемых образцов при больших обжатиях абсолютные значения предела прочности (максимального механического напряжения, выше которого происходит разрушение материала), полученные при испытаниях на разрыв, выше в проволоке деформированной волокой со сдвигом в сравнении с проволокой, изготовленной по стандартной технологии (для круглой волоки ф 4.65 предел прочности равен $\sigma=1210 \mathrm{~N} / \mathrm{mm}^{2}$, а для волоки со сдвигом $\varnothing 4.65 \sigma=1280 \mathrm{~N} / \mathrm{mm}^{2}$ [7]). Заметим, что для стали 08Г2С регламентируемый предел прочности составляет $1090-1280 \mathrm{~N} / \mathrm{mm}^{2}$. 


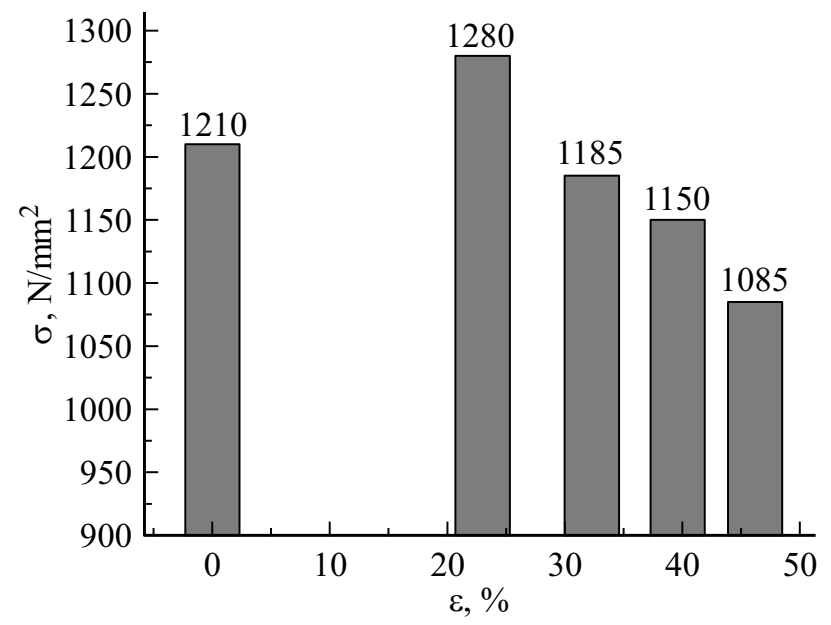

Рис. 2. Предел прочности проволоки после обжатия волокой со сдвигом $\varepsilon=23 \%$ и последующих степенях обжатия обычным волочением.

С увеличением суммарной степени обжатия волокой со сдвигом и круглой волокой $(32.3,39.6,46.2 \%)$ снижается прирост предела прочности (уменьшается для образцов № $2 \rightarrow$ № 5, рис. 2). Это - необычное явление, так как увеличение степени деформации (обжатия) должно приводить к большему росту прочности. Поэтому важную роль играли исследования пластичности [9], где был проведен анализ поперечного сечения образцов. Исследования показали, что применение сдвиговой волоки приводит к росту относительного сужения для всех вариантов обжатий. Относительное сужение с ростом степени обжатия сдвиговой волокой существенно увеличивается. Например, для образца № 1, $\varnothing 5.57$ в исходном состоянии относительное сужение $\psi=50 \%$, применение же сдвиговой волоки для образца № 2, ф4.65 способствовало увеличению относительного сужения до $\psi=75 \%$ [9]. Это благоприятный факт, так как относительное сужение тесно связано с технической пластичностью проволоки во время волочения.

\section{2. Неравномерное увеличение плотности с ростом степени обжатия}

Для уточнения природы роста относительного сужения с увеличением степени деформации были проведены измерения плотности. Как видно из рис. 3, для режима волочения „сдвиговая волока $(23 \%)+$ круглая волока $(9-23 \%)^{،}$ плотность неравномерно возрастает с увеличением суммарной деформации; значения плотности наибольшие, $7.767 \mathrm{~g} / \mathrm{cm}^{3}$ для образца № 5 .

Полученный результат показывает, что после деформации в режиме „сдвиговая волока + круглая волока“ плотность для всех случаев обжатий увеличивается, означая, что смена сдвиговой волоки на круглую приводит к залечиванию пор за счет изменения направления течения металла. Этот результат является дополнитель- ным благоприятным фактором для повышения технологической пластичности при волочении со сдвигом.

\section{3. Перестраивание микроструктуры при различных деформациях}

Рассмотрим подробно изменения зеренной структуры при разных режимах деформации, выявленные с помощью растровой микроскопии проволок в поперечном сечении (рис. 4). Из представленных картин следует, что с ростом степени обжатия при волочении сдвиговой волокой микроструктура становится более мелкой: ферритные зерна уменьшаются, перлитные колонии имеют в 1.5 раза меньший размер, цементитные пластины раздроблены и однороднее распределены по ферриту внутри перлитных колоний. Изменение структуры является ожидаемым, так как большие частичные обжатия сдвиговой волоки и должны приводить к измельчению структуры. Наиболее мелкая и однородная структура возникает, когда обжатия составляют $(23+9.3) \%$ (рис. $4, c)$ : не только измельчается зерно, но и внутри него формируется большая плотность субграниц. Данные факты являются важным доказательством того, что волочение со сдвигом может приводить не только к последовательному уменьшению размера зерен и, как следствие, к росту прочности с падением пластичности, но и к увеличению роста зерна и снижению темпа роста прочности с ростом пластичности, что объясняется развитием динамической полигонизации [7], приводящей и к росту зерен, из-за перемещения границ зерен и аннигиляции пор.

C практической точки зрения этот факт важен, так как процесс волочения проволоки можно не прекращать из-за отсутствия порывов благодаря залечиванию пор, источников зарождения микротрещин с последующим опасным развитием магистральной трещины и разру-

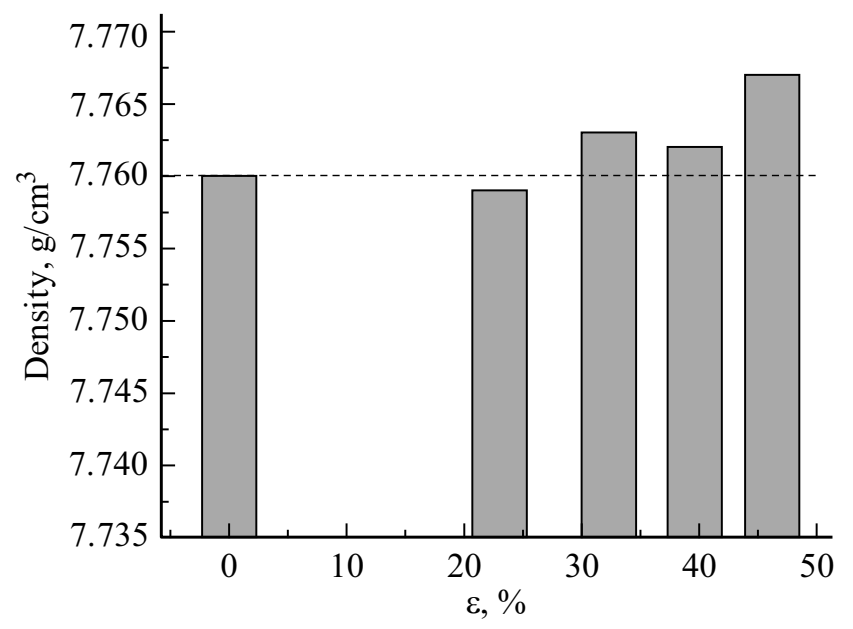

Рис. 3. Результаты измерения плотности образцов № $1 \rightarrow$ № 5 после обжатия волокой со сдвигом $23 \%$ и разных степенях последующего обжатия обычным волочением. 

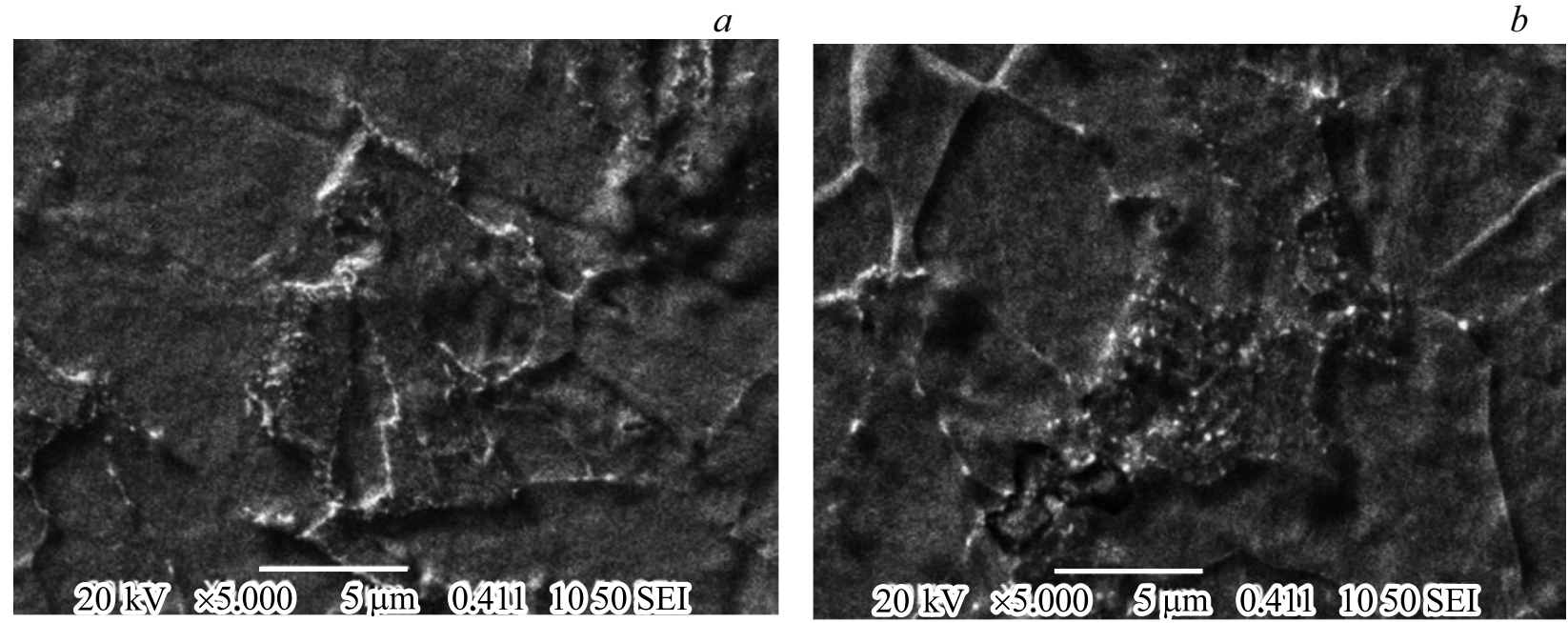

$c$

$d$
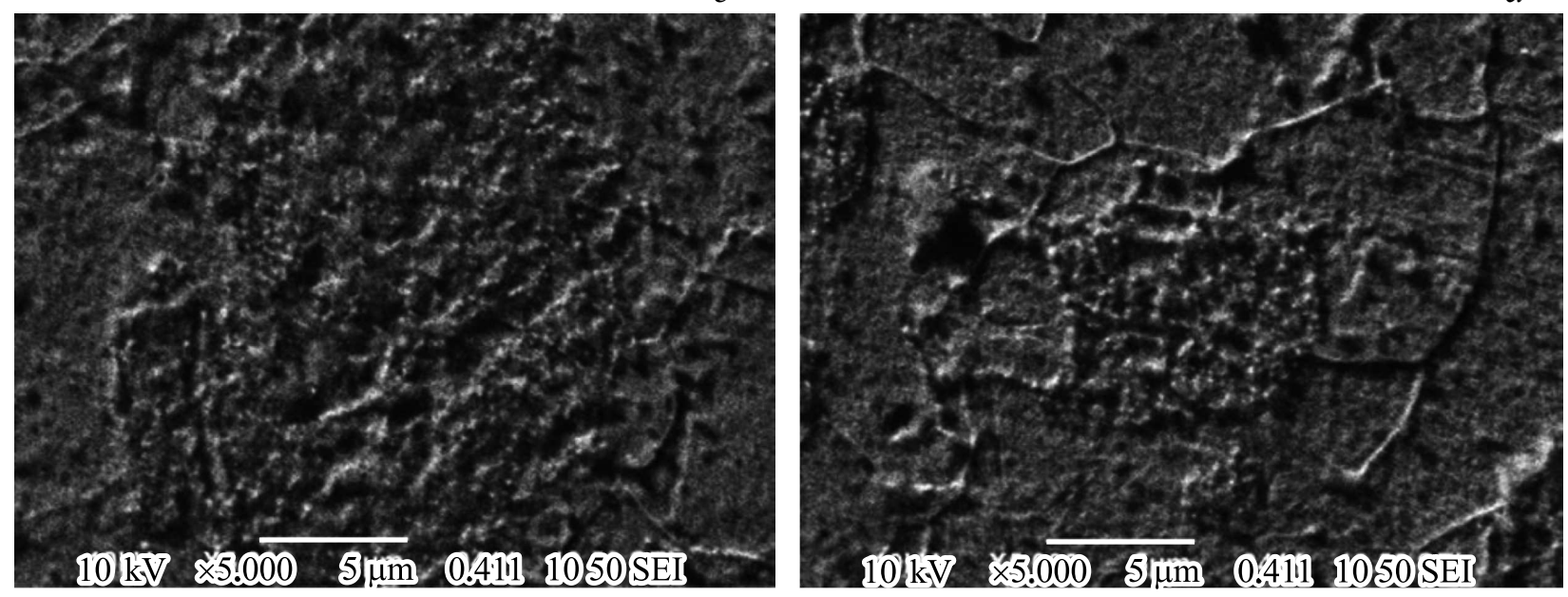

$e$

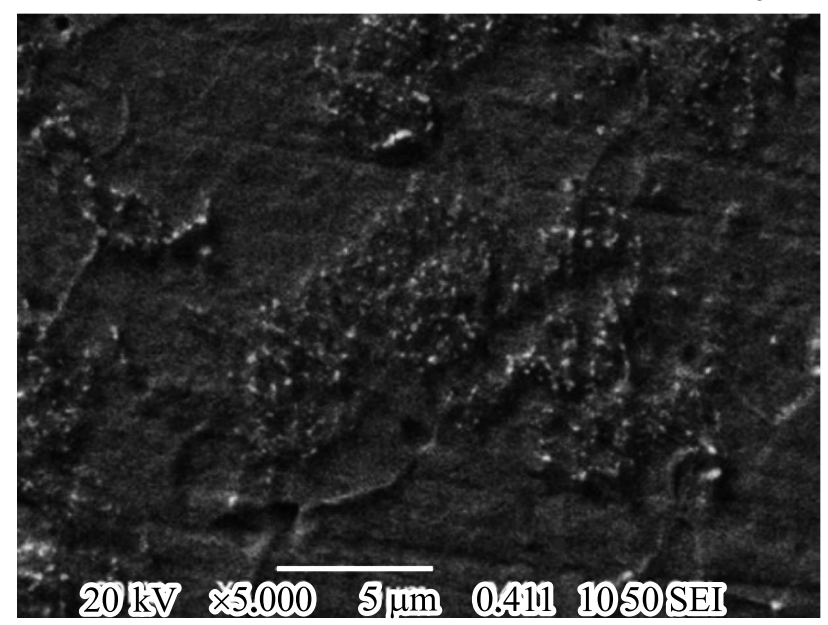

Рис. 4. Микроструктура поперечного сечения образцов стали после волочения через круглую волоку до $\varnothing 5.57(a)$, волочения со сдвигом до $\varnothing 4.65(b)$ и последующего волочения через круглую волоку $\varnothing 4.65(c), \varnothing 4.46(d), \varnothing 4.28(e)$. Соответствующие деформации указаны на рисунке: $a$ - № $1, \varepsilon=0 ; b-$ № $2, \varepsilon=23 \% ; c-$ № $3, \varepsilon=32.3 \% ; d-$ № 4 , $\varepsilon=39.6 \%$; $e-$ № 5 , $\varepsilon=46 \%$.

шения, о чем предостерегает В.Л. Гиляров [19]. Эти эффекты позволяют реализовать повышение пластич- ности проволоки без отжига в процессе волочения до технически необходимого уровня. 

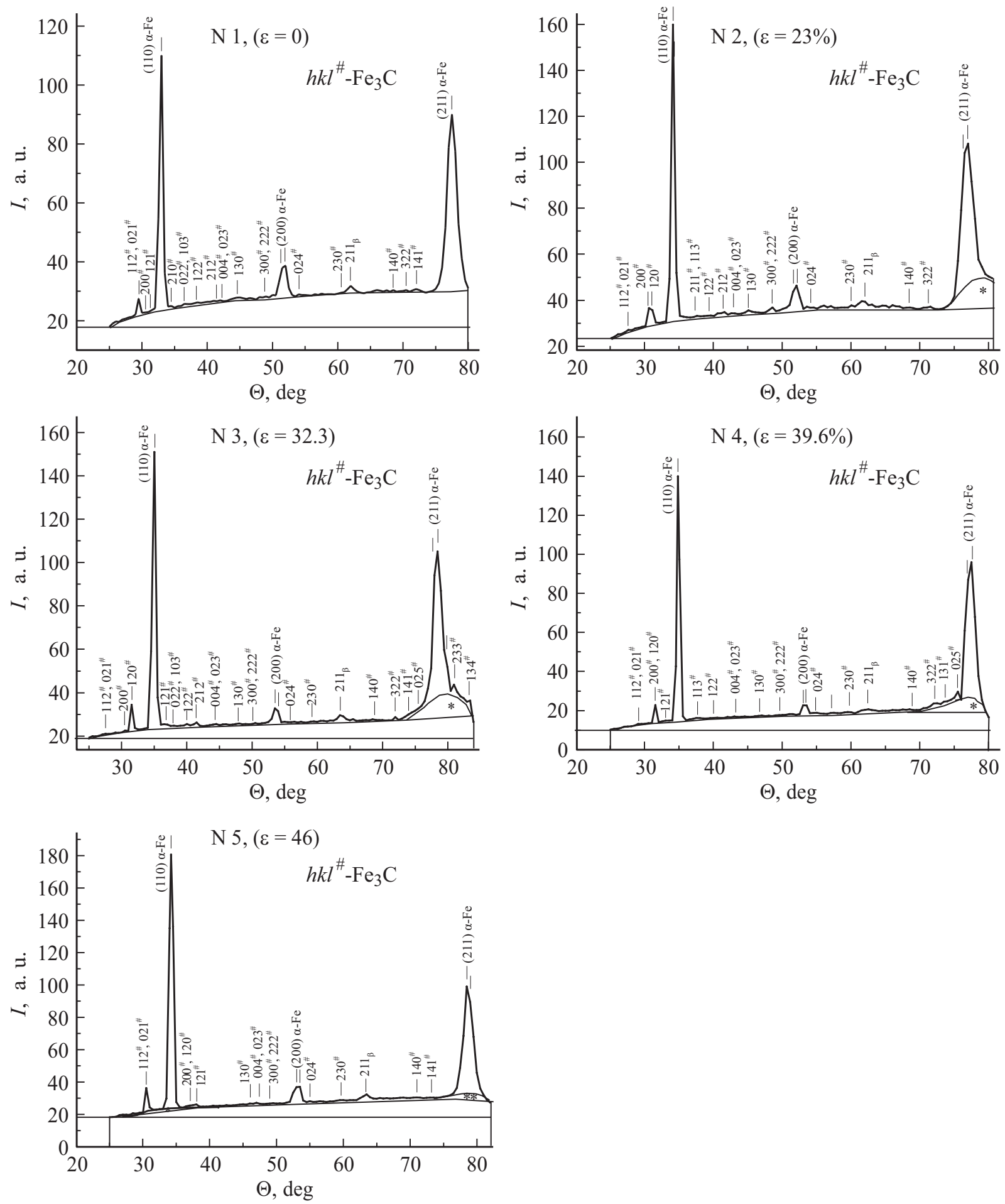

Рис. 5. Дифракционная картина от поперечного сечения образца № 1 стали $08 Г 2 С$, деформация $\varepsilon=0$. Гало отмечено звездочкой.

\section{4. Воздействие деформации волочением со сдвигом на атомный порядок стали}

Проследим за особенностями различных вариантов деформационного воздействия на связь изменений механических свойств и атомного порядка. На дифракционной картине от поперечного сечения образца № 1 (рис. 5) видны яркие дебаевские линии, соответствующие кубической ОЦК-решетке поликристаллической структуры.

Линия от плоскостей (110) имеет максимальную интенсивность, узкая, симметричная, что свидетельствует о высоком уровне атомного порядка в данной группе плоскостей, проходящих через центральный атом кри- 
сталлической ячейки и имеющих наибольшую заселенность среди других типов плоскостей ОЦК-решетки.

Две другие линии от плоскостей (200) и (211) увеличены по ширине, при этом (200) имеет плоскую вершину, что означает присутствие в указанных семействах параллельных плоскостей подгрупп однотипных плоскостей, сжатых или растянутых относительно друг друга.

Над линией фона выявляются диффузные максимумы слабой интенсивности, что указывает на присутствие упорядоченных мелких атомных группировок в сильно разупорядоченной деформированной структуре материала стали. Как можно видеть на рис. 5, диффузные максимумы принадлежат карбидной фазе $\mathrm{Fe}(\mathrm{Mn})_{3} \mathrm{C}$.

Полученные результаты позволяют отметить проявление разномасштабной предпочтительности атомного порядка присутствующих в стали фаз: металлической фазы, $\alpha$-Fe, в виде поликристаллической структуры с дальним атомным порядком, и карбидной фазы, $\mathrm{Fe}(\mathrm{Mn})_{3} \mathrm{C}$, в форме наноразмерных кластеризованных атомных группировок, „втертых в пластичную структуру матрицы, но сохраняющих свою кристаллографическую индивидуальность“, и потому проявляющихся над фоном в виде уплощенных максимумов слабой интенсивности, но с хорошо различимыми положениями дифракционных углов $\Theta$, характерных для карбидной фазы (рис. 5).

Дебаевские максимумы основной $\alpha$-Fe-фазы с дальним атомным порядком образца № 2 имеют плоскую или раздвоенную вершину, кроме отражения (110), которое имеет вид узкой $\delta$-функции, что характеризует состояние структуры плоскостей (110), как наиболее релаксированное и совершенное (рис. 5, образец № 2).

Для других плоскостей $\alpha$-Fe-фазы, (200) и (211), сложная форма их интерференционных вершин свидетельствует о расслоении в семействах указанных плоскостей на сжатые и растянутые группы плоскостей, т.е. имеем эффект продольной волны в материале образца № 2 .

Дебаевские линии от семейств плоскостей (200) и (211), $\Theta>50^{\circ}$, основной фазы $\alpha$-Fe, имеют необычную форму (рис. 5, образец № 3). Они имеют вид групповых максимумов, что означает присутствие в структуре дальнего порядка мезоскопических искажений, включающих десятки и даже сотни плоскостей соответствующего типа в виде целых областей структуры материала сжатых или растянутых (по сравнению с релаксированным исходным состоянием).

Такое локально расслоенное состояние напряженных, однако сохраняющихся в материале, указанных групп плоскостей выявляется в сопровождении аморфизированной фракции из плоскостей (211) с нарушенным дальним атомным порядком, но имеющих его в виде наноразмерных атомных группировок (кластеров). На рис. 5 аморфизированная фракция представлена в виде диффузного максимума-гало у основания линии (211).

Дебаевская линия от семейства плоскостей (211) асимметричная, со стороны больших углов $\Theta$ расщеп- ленная (рис. 5, образец № 4), у основания - наплыв с несколькими диффузными максимумами, в основании линии - диффузная полоса гало с максимумом, соответствующим угловому положению максимума линии (211) и затянутым спадом интенсивности вплоть до линии (200), плоская вершина которой указывает на формирование в данной группе идентичных плоскостей типа (200), но с широким набором межплоскостных расстояний.

Основные изменения дифракционной картины образца № 5 (рис. 5, образец № 5) дополняют описанные эффекты для образцов № 2-4. Форма линий (211) и (200) означает формирование в структуре этих плоскостей критических нарушений атомного порядка с фазовым переходом (ФП) второго рода типа порядок $\rightarrow$ беспорядок, который наглядно проявляется в виде формирования аморфной фракции в поликристаллической основной структуре (гало на рис. 5). Этот ФП привел к частичной релаксации упругих напряжений в деформированной структуре, что подтверждается слабой интенсивностью флуктуаций диффузного когерентного рассеяния, имеющих вид размытых максимумов карбидной фазы $\mathrm{Fe}_{3} \mathrm{C}$ над линией фона (рис. 5).

\section{5. Расслоение кристаллографических плоскостей (211) на кластерные группы}

В результате анализа формы наиболее структурно чувствительного дифракционного максимума от плоскостей (211) на рис. 5 видно, что от симметричной дельтаобразной функции для отожженного состояния в случае деформированных образцов форма максимума (211) непрерывно меняется, расщепляясь в области вершины и образуя дополнительный максимум-гало

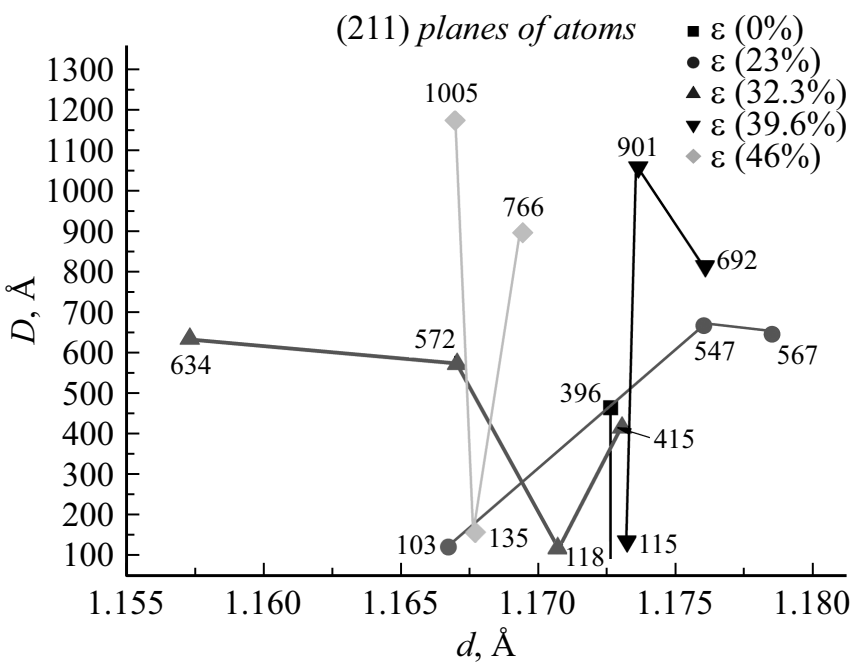

Рис. 6. Структурные группы „сжатых и растянутых“ плоскостей (211), а также аморфизированных фрагментов плоскостей этого типа на рис. 6 нижние точки для гало у основания линии (211) по рис. 5, сформированных в результате применения последовательно усиливающейся деформации, $\varepsilon=0-46 \%$. 
у основания линии. Проведенные нами исследования выявленных деталей тонкой структуры дифракционных картин представлены на рис. 6.

Расщепление линии (211) указывает на эффект пространственного расслоения однотипных кристаллографических плоскостей (211) на группы, различающиеся величиной межплоскостного расстояния $(d)$, линейным размером $(D)$ атомных группировок (кластеров), количеством плоскостей в группировке $(n)$ и характером расположения образовавшихся групп относительно ненапряженного релаксированного состояния.

Величина $d$ определяется по справочным таблицам для экспериментально измеренных дифракционных углов $\theta$ выявленных локальных максимумов в $\mathrm{Cr} \mathrm{K} \alpha$ излучении; линейный размер $D$ группировок плоскостей или их фрагментов (последние относятся к случаю аморфных групп) рассчитан по измеренной полуширине соответствующих диффузных максимумов по методике Китайгородского [18]; количество плоскостей в группировке (на рис. 6 написано цифрами $103, \ldots, 1005$ рядом с символами для соответствующих образцов) оценивалось по формуле для диска $n=D / d$.

\section{6. Самоорганизация атомного порядка}

Учитывая, что в сильно искаженных структурах интенсивность как когерентного, так и некогерентного диффузного рассеяния пропорциональна числу рассеивающих центров, оценили долю порядка и беспорядка в расположении атомов по их интегральным интенсивностям: $I_{\text {coh }}+I_{\text {incoh }}=100 \%$ (рис. 7). Из представленных на рис. 7 зависимостей следует, что в материале сильнодеформированных образцов с $\varepsilon_{\mathrm{N} 1}=0 \%$ и $\varepsilon_{\mathrm{N} 2}=23 \%$, даже несмотря на релаксационный отжиг образца № 1, в пространственном расположении атомов преобладает беспорядок над порядком, о чем свидетельствует более высокая интегральная интенсивность некогерентно-

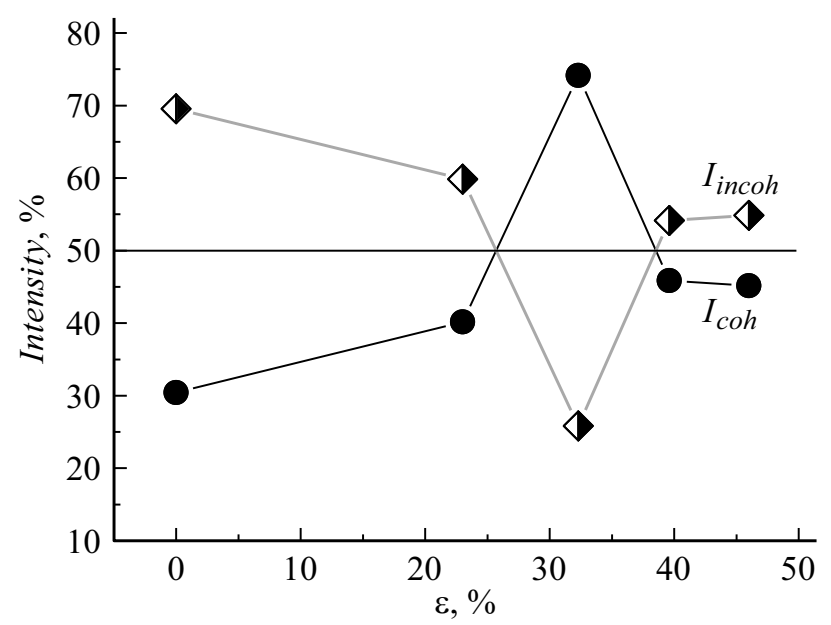

Рис. 7. Изменение интенсивностей когерентного и некогерентного рассеяний рентгеновских лучей образцами стали 08Г2С в зависимости от деформации.

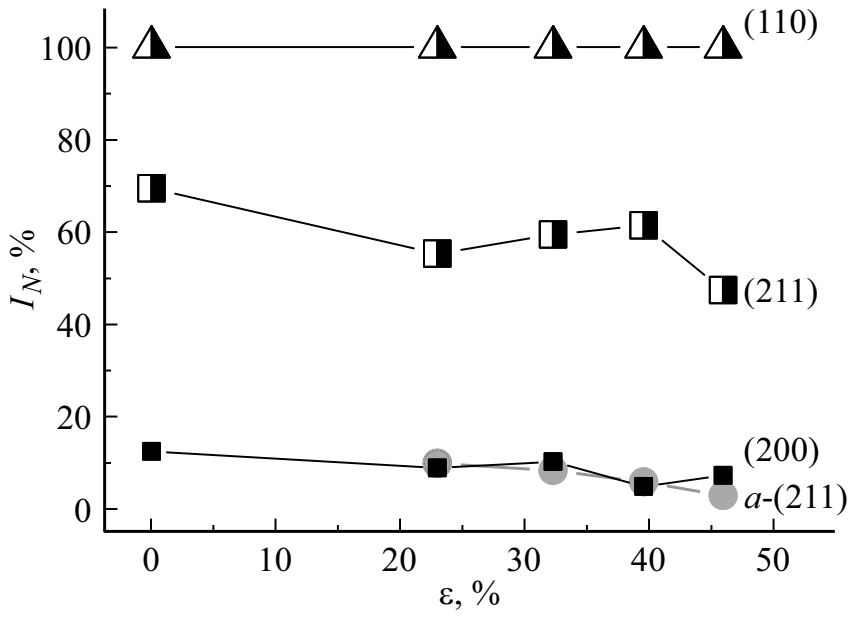

Рис. 8. Изменение интенсивностей когерентного рассеяния рентгеновских лучей от различных кристаллографических структурных групп в образцах стали 08Г2С в зависимости от деформации; от аморфных кластеров символы обозначены $a$ (211).

го рассеяния по сравнению с когерентным для этих образцов.

Однако в образце № 3 при увеличении суммарной деформации до $\varepsilon_{\mathrm{N} 3}=32.3 \%$ произошел структурный переход второго рода типа беспорядок $\rightarrow$ порядок и при дальнейшем увеличении деформации образцов $\varepsilon_{\mathrm{N} 4}=39.6 \%$ и $\varepsilon_{\mathrm{N} 5}=46 \%$ интенсивности когерентного и некогерентного рассеянных излучений близки по величине: $I_{\text {coh }}: I_{\text {incoh }}=45: 55 \%$. Данные результаты показывают развитие процессов самоорганизации в многоатомной конденсированной системе, подвергаемой сильному механическому воздействию.

При нормировании на 100 единиц интенсивности главного отражения (110) ОЦК-фазы железа для каждой из дифракционных картин исследуемых образцов сравнили характер изменений нормированных интенсивностей когерентного рассеяния семействами других плоскостей (211) и (200), а также от аморфизированных групп $a$ (211) на рис. 8.

Результаты рис. 8 показывают, что по сравнению с исходным релаксированным состоянием $(\varepsilon=0)$ для образцов № $2 \rightarrow$ № 5 интенсивности линий от плоскостей (211) и (200) нелинейно уменьшаются (211) для № 3 и № 4 сначала увеличивается, а затем для № 5 вновь уменьшается, приближаясь к 40\%, по сравнению с 70\% для исходного образца; (200) уменьшает интенсивность, флуктуируя от 12\% для № 1 до $8 \%$ для № 4; аморфная группа $a$-(211) наиболее интенсивная $11 \%$ появляется впервые для образца № 2, затем постепенно уменьшается по интенсивности до 3\% для № 5 .

Полученные результаты показывают стремление системы „гасить“, а не „раскачивать“ нарушение порядка, о чем свидетельствует, например, группа антисимметрично расположенных точек линий (211) и (200) для деформаций $(\varepsilon=32.3 \rightarrow 39.6 \rightarrow 46 \%)$ на рис. 8 . 


\section{Заключение}

1. Выявлен эффект пространственного расслоения однотипных кристаллографических плоскостей (211) на кластерные группы, различающиеся величиной межплоскостного расстояния в пределах $1.156<d_{0}=$ $=1.172<1.178 \AA$ с линейным размером группировок $D=100-1100 \AA$, количеством плоскостей в группировке $n=100-1000$ и характером расположения образовавшихся групп относительно ненапряженного релаксированного состояния $(\varepsilon=0)-d_{0}=1.172 \AA, D=450 \AA$, $n=396$. При этом наибольший разброс плоскостей по величине межплоскостных расстояний реализовался в образцах № $2(\varepsilon=23.2 \%)$ и № $3(\varepsilon=32.3 \%)$, $d=(1.157-1.177) \AA$, а по величине размеров группировок - в образцах № $4(\varepsilon=39.6 \%)$ и № $5(\varepsilon=46 \%)$ соответственно $D_{\mathrm{N} 4}=100-1100 \AA . D_{\mathrm{N} 5}=100-1200 \AA$.

2. Обнаружен ФП порядок $\rightarrow$ беспорядок $\rightarrow$ новый модифицированный порядок с локальным разрушением дальнего атомного порядка в семействе плоскостей (211) и образованием аморфизированной фракции кластерной фазы из фрагментов разрушенных плоскостей этого типа. Аморфной структурной фракции нет в исходном отожженном состоянии, ее концентрация уменьшается до предела чувствительности метода, 3\%, в образце с наибольшей деформацией № 5 ( $\varepsilon=46 \%)$.

3. Замечен и проанализирован характер изменения симметрии распределения структурных групп относительно исходного углового положения дифракционного максимума от плоскостей (211). В исходном состоянии для образца № $1(\varepsilon=0)$ имеем максимум в виде симметричной дельтаобразной функции с $d_{0}=1.172 \AA$. По мере увеличения деформации образцов сначала в № $2(\varepsilon=23.2 \%)$ и № $3(\varepsilon=32.3 \%)$ наблюдается асимметричное распределение новых образований относительно исходного: два справа и один слева (№ 2) или три слева и один справа (№ 3). Затем же для образцов № $4(\varepsilon=39.6 \%)$ и № $5(\varepsilon=46 \%)$ полностью целая структурная группа образца располагается то справа (№ 4), то слева (№ 5) относительно положения для состояния исходного образца. Полученный результат указывает, что для сильно деформированного материала энергетически выгодной является предпочтительность разномасштабной фрагментации атомной структуры при компактировании групп плоскостей либо в состоянии растяжения, при деформации образца $(\varepsilon=39.6 \%)$, либо же в состоянии сжатия, при деформации $(\varepsilon=46 \%)$.

\section{Список литературы}

[1] Valiev R.Z., Langdon T.G. // Progr. Mater. Sci. 2006. Vol. 51. N 7. P. 881-981.

[2] Beygelzimer Y.E., Varyukhin V.N., Orlov D.V., Synkov S.G. Twist Extrusion - the accumulation of strain. Donetsk Company „TEAH“, 2003. P. 87.

[3] Zhilyaev A.P., Langdon T.G. // Progr. Mater. Sci. 2008. Vol. 53. N 6. P. 893-979.
[4] Valiev R.Z., Alexandrov I.V. Nanostructured materials produced by severe plastic deformation. M.: Logos, 2000. $272 \mathrm{p}$.

[5] Stepanov N.D., Kuznetsov A.V., Salishchev G.A., Raab G.I., Valiev R.Z. // Mater. Sci. Eng. A. 2012. Vol. 554. P. 105-115.

[6] Varyukhin V.M., Belousov N.N., Pashins'ka O.G., Tkachenko V.M. // Metallofizika i Noveishie Tekhnologii. 2005. Vol. 27. P. 1113-1119.

[7] Пашинская Е.Г. Физико-химические основы измельчения структуры при комбинированной пластической деформации. Донецк: Вебер, 2009. 352 с.

[8] Yu H.L., Lu C., Tieu A.K., Li H.J., Godbole A., Zhang S.H. // Advan. Engineer. Mater. 2016. Vol. 18. N 5. P. 754-769.

[9] Pahynskaya E.G., Myshlyayev M.M., Varyukhin V.N., Stolyarov V.V., Mironov S.A., Tkachenko V.M. // Bulletin of the Russian Academy of Sciences Physics. 2009. Vol. 73. N 9. P. 1319-1323.

[10] Hwang S.K., Baek H.M., Son I.H., Im Y.T., Bae C.M. // Mater. Sci. Eng. A. 2013. Vol. 579. P. 118-125.

[11] Lee J.W., Baek H.M., Hwang S.K., Son I.H., Bae C.M., Im Y.T. // Mater. Design. 2014. Vol. 55. P. 898-906.

[12] Pashinskaya E.H., Zavdoveev A.V., Maksakova A.A., Varyukhin V.N., Tolpa A.A., Tkachenko V.M. // High Pressure Physics and Technics. 2015. Vol. 25. P. 107-122.

[13] Pahynskaya E.G., Zavdoveev A., Mironov S., Varyukhin V.N., Maksakova A. // Intern. J. Mater. Research. 2016. Vol. 107. P. 239-244.

[14] Самойленко 3.А., Ивахненко Н.Н., Пушенко Е.И., Пашинская Е.Г., Варюхин В.Н. // ФТТ. 2014. Т. 56. Вып. 6. C. $1186-1190$.

[15] Самойленко 3.А., Ивахненко Н.Н., Пушенко Е.И., Пашинская Е.Г., Варюхин В.Н. // ФТТ. 2015. Т. 57. Вып. 1. С. 82 90.

[16] Самойленко 3.А., Ивахненко Н.Н., Пушенко Е.И., Пашинская Е.Г., Варюхин В.Н. // ФТТ. 2016. Т. 58. Вып. 2. С. 217 224.

[17] Кривоглаз М.A. Электронная теория гетерогенных состояний в твердых телах. М.: Наука, 1988. С. 3-39.

[18] Китайгородский Л.И. Рентгеноструктурный анализ мелкокристаллических и аморфных тел. М.: Наука, 1952. $588 \mathrm{c.}$

[19] Гиляров В.Л. // ФТТ. 2005. Т. 47. Вып. 5. С. 808-811. 